\title{
A New Electrostatic Generator Driven by Only an Electric Field of an Electret
}

\author{
Katsuo Sakai \\ Electrostatic Generator Laboratory, Yokohama, Japan \\ Email: gy7a-ski@asahi-net.or.jp
}

How to cite this paper: Sakai, K. (2021) A New Electrostatic Generator Driven by Only an Electric Field of an Electret. Journal of Electromagnetic Analysis and Applications 13, 161-171.

https://doiorg/10.4236/jemaa.2021.1312012

Received: August 24, 2021

Accepted: November 29, 2021

Published: December 2, 2021

Copyright $\odot 2021$ by author(s) and Scientific Research Publishing Inc. This work is licensed under the Creative Commons Attribution International License (CC BY 4.0).

http://creativecommons.org/licenses/by/4.0/

\section{(c) (i) Open Access}

\begin{abstract}
Today, the sun is a very useful source of energy because it continuously radiates energy. An electron is radiating energy continuously, too. A new electrostatic generating method using this electric field energy from electrons as a driving force of charge carriers was invented, and its success was presented on ESA 2017 and ESA 2019. This new electrostatic generator was realized by asymmetric electrostatic force, which is a new phenomenon. Unfortunately, its experimental success rate was rare. Therefore, the cause was searched by many experiments. Finally, it became apparent that the acceleration force of the charge carrier was not stronger than the deceleration force of the charge carrier with this experimental equipment. Therefore, the electrode arrangement of this equipment was improved. As a result, the acceleration force was increased, and the deceleration force was decreased. Then, the experimental success rate became $100 \%$.
\end{abstract}

\section{Keywords}

Electrostatic Generator, Electret, Asymmetric Electrostatic Force,

Charge Carrier, Asymmetric Shape Charged Cond uctor

\section{Introduction}

1) Two very useful so urces of energy: sun and electron

Today, the very useful so urce of energy to solve environmental problems is the sun, because it continuously radiates a large amount of energy. Like the sun, an electron also radiates energy around it continuously. Of course, the radiated energy from an electron is very little. However, the number of electrons in our world is huge. An electret keeps electrons on its surface for a very long time (100 years). Therefore, if a new electrostatic generator is driven by an electret only, it will solve the environmental problem. Because it does not produce $\mathrm{CO}_{2}$ and it is 
safe. Electrets also produce electric energy continuously, which have a long lifetime, and low cost.

\section{2) Asymmetric electrostatic force}

For a long time, the electrostatic force has been calculated by the well-known Coulomb's Formula (1). It is apparent from this formula that the magnitude of this electrostatic force does not change when the direction of the electric field turns over.

$$
f=q E
$$

where $f$. Electrostatic force that acts on a point charge.

$q$ : Quantity of a point charge;

$E$ : The intensity of the electric field in which a point charge is placed;

The application of this formula is limited to point charges and sphere-shaped charge carriers [1].

A new electrostatic generator driven by only an electric field using Coulomb's law was tried for several years. Many different ideas were tested by simulation and simple experiments. However, no solution was found, but a very useful phenomenon was found in those simulations by chance. A new electrostatic generator driven by this new phenomenon was invented.

The electrostatic force that acts on an asymmetric charged conductor changes largely when the direction of the electric field reverses, as shown in Figure 1. This new phenomenon was named asymmetric electrostatic force [2]-[8]. The electric field on the left side of Figure 1 was named a forward electric field, and the electric field on the right side was named a backward electric field.

\section{3) Basic the ory of the new electrostatic generator}

The basic theory of an electrostatic generator is defined by lifting the charge to a high potential by mechanical force against the electric force that acts on this charge. It is impossible for the mechanical force to carry the charge directly. Therefore, the charge is packed into a suitable body. We call this body the charge carrier. A basic unit of the new electrostatic generator that is driven by asymmetric electrostatic force is concretely shown in Fig ure 2.

This generator mainly consists of a charge injection electrode, driving electrode, charge collection electrode, and charge carrier.

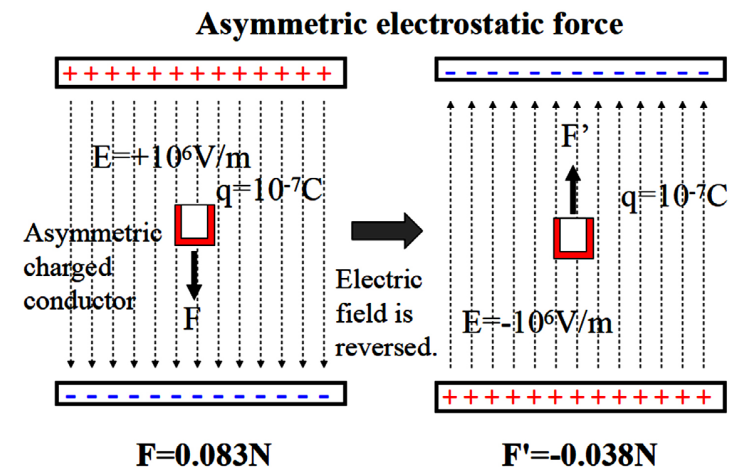

Figure 1. Electrostatic force that acts on charged box conductor (asymmetric electrostatic force). 


\section{$\mathrm{Fe} 1>\mathrm{Fe} 2$}

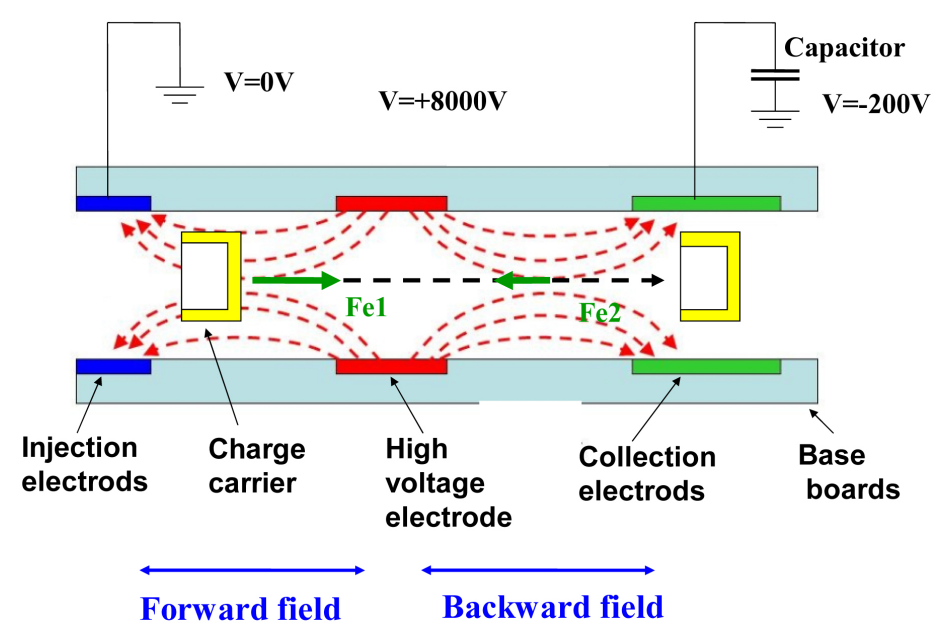

Figure 2. Schematic layout of one unit of the new electrostatic generator.

A positive high voltage was applied to the driving electrode. The injection electrode was grounded. The collection electrodes were kept at a negative low voltage. As a result, the driving electrode and the injection electrode produced a forward electric field for a negative charge between them. The driving electrode and the collection electrode produced a backward electric field for a negative charge between them.

A gutter-shaped conductor was used as a charge carrier that carries a negative charge (electron) from the injection electrode to the collection electrode through the driving electrode.

The asymmetric electrostatic phenomenon produces a large electrostatic force $\mathrm{Fe} 1$ in the forward electric field and a weak electrostatic force Fe2 in the backward electric field. Therefore, the charge carrier gains large kinetic energy in the forward electric field. Then, it loses some of its kinetic energy in the backward electric field. As a result, the charge carrier maintains extra kinetic energy when it arrives at the collection electrode. The carried charge can be lifted to a higher potential by this extra energy.

This is the principle of the electric field-driven generator.

4) Simulation results of the energy gained using the new electrostatic generator

Now that the basic principle of the new electrostatic generator has been made clear; the electric energy gained by the smallest unit of the new generator can be simulated [2] [3].

When the width of a gutter-shaped charge carrier is $80 \mu \mathrm{m}$, the width of a driving electrode is $120 \mu \mathrm{m}$ and the distance between electrodes is $320 \mu \mathrm{m}$. The electrostatic force that acts on the charge carrier was simulated.

The two-dimensional finite difference method was used to simulate this electrostatic force.

Figure 3 shows the results of the simulation. 


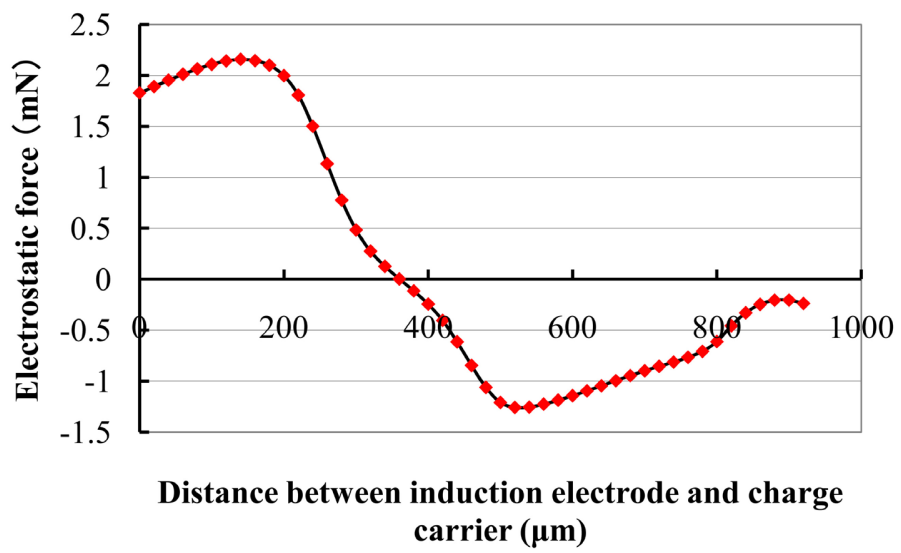

Figure 3. Electrostatic force as a function of the distance from the injection electrode to the charge carrier.

The electrostatic force in the forward electric field $(0-380 \mu \mathrm{m})$ is positive; therefore, the charge carrier gains kinetic energy. In contrast, it becomes negative in the backward electric field $(380-920 \mu \mathrm{m})$. Therefore, the charge carrier loses some of its kinetic energy. As a result, the charge carrier maintains extra kinetic energy, 1.53E-7 J, when it arrives between the collection electrode. The carried charge quantity is $-5.86 \mathrm{E}-10 \mathrm{C}$. This charge can be lifted to a higher potential by extra energy. This possible potential is $-261 \mathrm{~V}$.

Therefore, when the charge carrier transfers from the injection electrode to the collection electrode, the generated electric energy becomes 1.37E-7 J.

5) Experimental equipment of the electric field-driven generator

Figure 4 shows the front view, and Figure 5 shows a plane view of the experimental equipment.

This equipment mainly consists of a charge injection electrode, a driving electrode, a charge collection electrode, and a charge carrier disk that has six charge carriers.

The charge carrier disk is a $0.5-\mathrm{mm}$ thick PET plate with a diameter of $95 \mathrm{~mm}$. ADB (Autonomous Decentralized Bearing) bearings were fixed on the center of the disk.

The six charge carriers were placed at 60-degree intervals, as shown in Figure 6.

The two charge injection electrodes, the two driving electrodes, and the two charge collection electrodes were placed on the main PET base plate at 60-degree intervals, as shown in Figure 6. The injection electrode was always grounded, the driving electrode was connected to a high voltage power supply, and the surface potential of the collection capacitor was measured by a surface potential meter.

The collection electrodes could perform semi-Faraday gauges. When the charge carrier was connected to the collection electrode by the aluminum foil, more than $90 \%$ charge on the charge carrier was transferred to the collection electrode (simulation result). 


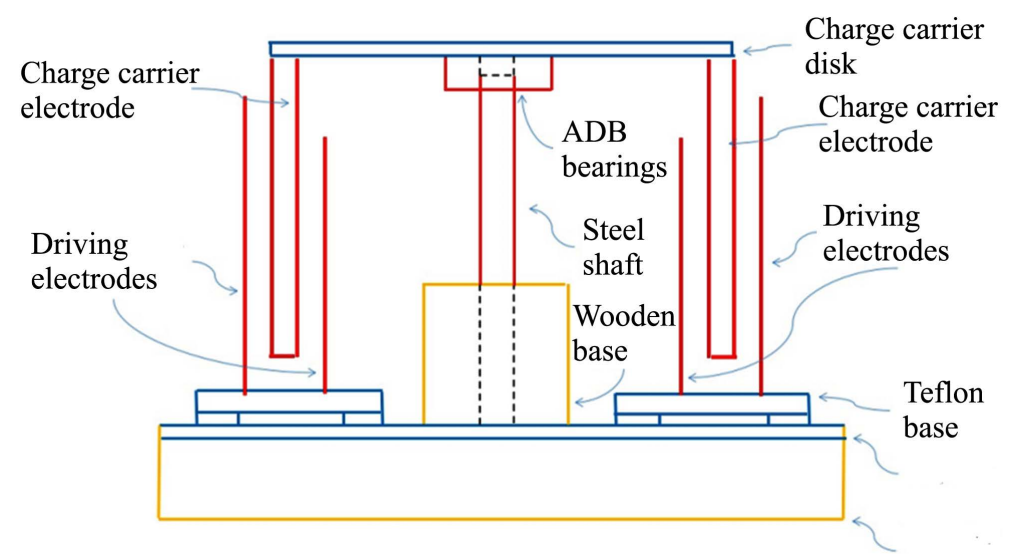

Figure 4. Front view of the experimental equipment of the electric field driven generator.

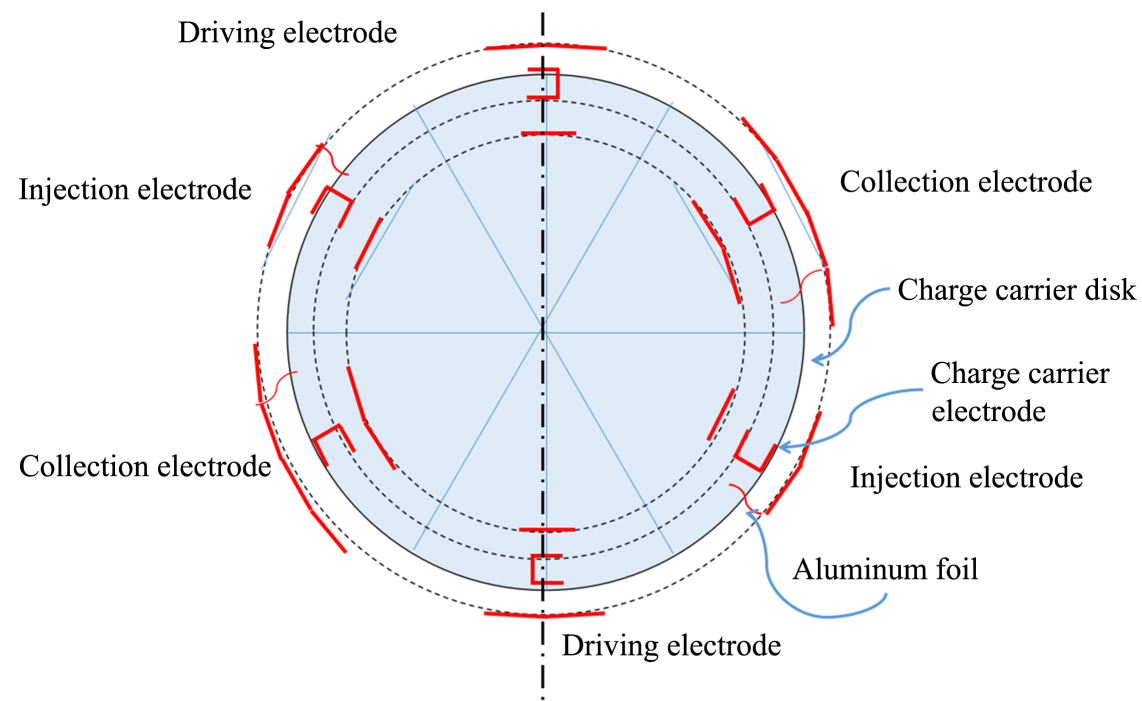

Figure 5. Plane view of the experimental equipment of the electric field driven generator.

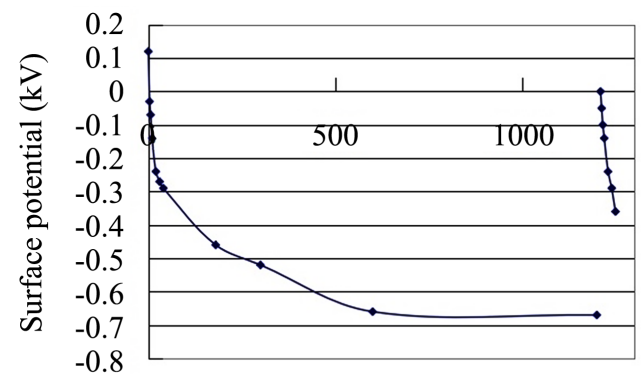

Rotation time of charge carrier di sk (Seconds)

Figure 6. The surface potential change of the capacitor of the collection electrode for the rotation time of the charge carrier disk.

A surface potential meter (SHISHIDO ELECTROSTATIC: STATIRON-DZ 3) was used to measure the surface potential of the collection capacitor.

6) Experimental result of the electric field-driven generator

After a high voltage was applied to the driving electrode, the charge carrier disk 
was rotated by an airflow. After the airflow was stopped, the charge carrier disk continued to rotate.

This result indicates that the charge carrier disk can rotate endlessly by the electrostatic force against the air resistance force and kinetic friction force.

When the charge carrier continues to rotate, the surface potential of the collection electrode capacitor becomes higher in the negative direction. These results mean that this experimental equipment continued to endlessly generate electric power.

Figure 6 shows the surface potential change of the collection electrode capacitor when the driving voltage was $8.2 \mathrm{kV}$.

This result was presented in ESA 2017 [9] [10], and an improved result was presented in ESA 2019 [11] using the new charging method shown in Figure 7.

In Figure 7, the upper and lower backplates of the charge carrier and the injection electrode temporarily produce two capacitors. Therefore, many injection charges (electrons) are injected into the back plates from the ground through the electric connector.

After the ESA 2019 conference, this experimental equipment was used repeatedly to replace the driving electrode with a driving electret.

At first, the same experiment equipment was used. However, the charge carrier disk did not rotate continuously. Many experiments were executed over one year, and the disk rotated continuously only a few times.

Therefore, the subject of this paper was to solve this problem.

\section{Analysis of the Problem and Its Resolution Result}

\section{1) Mea surement equipment of the real electrostatic force}

The simulation was performed with a straight line arrangement of the three electrodes, as shown in Fig ure 2; in contrast, in real equipment, the three electrodes were placed on circumference, as shown in Fig ure 5.

Therefore, it was estimated that the real electrostatic force that acts on the charge carrier will be weaker than the simulated electrostatic force.

Unfortunately, the homemade simulation program cannot simulate the real electrostatic force in the experimental equipment.

Therefore, the real electrostatic force was measured on the experimental equipment.

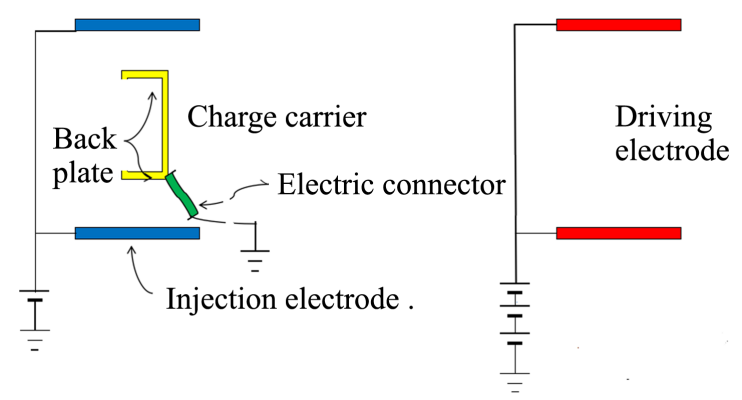

Figure 7. The improved charge injection method. 
The measurement of the real electrostatic force that acts on the charge carrier is very difficult because that force is very weak. Therefore, the free rotation time of the charge carrier disk after the disk was forced to rotate by air flow was measured. If the real electrostatic force is stronger than the air resistance force, the disk rotates for a long time. In contrast, if the real electrostatic force is weaker than the air resistance force, the free rotation time becomes short.

Figure 8 shows the plane view of the real electrostatic force measurement equipment.

The charge carrier is injected with a charge when it passes through the injection electrodes. The charge on the charge carrier is then released when the charge carrier enters the collection electrodes.

Two earth electrodes were placed before and after the driving electrode. The former was named the front earth electrode, and the latter was named the back earth electrode.

When the polarity of the charge on the charge carrier is negative and the polarity of the voltage of the driving electrode is negative, the charge carrier is decelerated between the front earth electrode and the driving electrode. The charge carrier is accelerated between the driving electrode and the back earth electrode.

The widths of the injection electrode, the driving electrode, and the two earth electrodes were $10 \mathrm{~mm}$. In addition, the width of the collection electrode was 15 $\mathrm{mm}$. The distance between the driving electrode and the two earth electrodes was $15 \mathrm{~mm}$.

The distance between electrodes, the voltage of the injection electrode and the driving electrode, the polarity of the voltage of the injection electrode and the width of the driving electrode were changed, and the free rotation time of the charge carrier disk was measured.

\section{2) The distance between the driving electrode and the earth electrodes}

Figure 9 shows the free rotation time of the charge carrier disk when the distance between the front earth electrode and the driving electrode was changed.

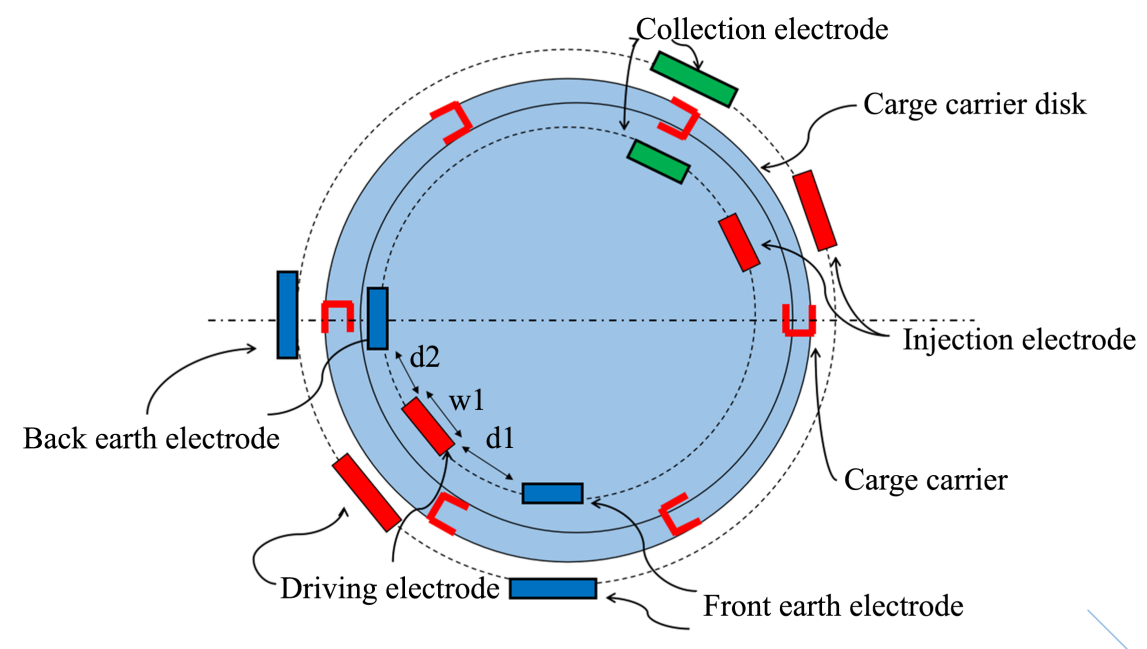

Figure 8. Real electrostatic force measurement equipment. 


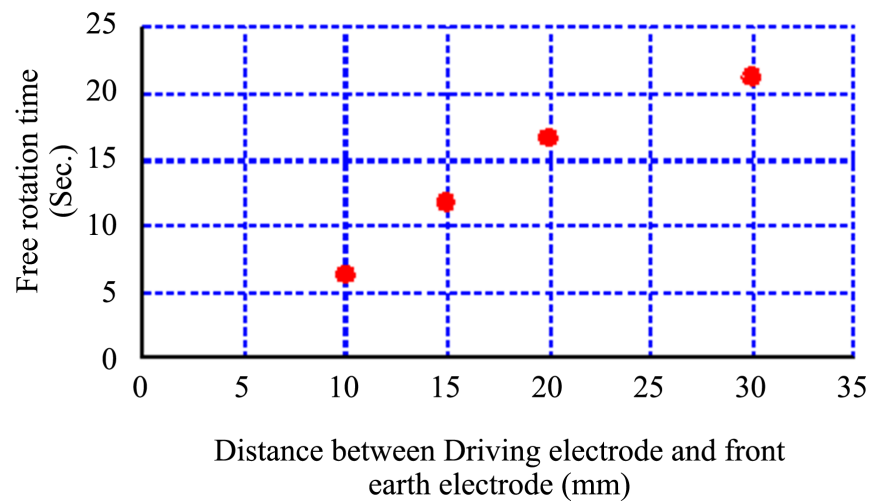

Figure 9. Free rotation time of the charge carrier disk when the distance between the front earth electrode and the driving electrode was changed.

It is apparent from Figure 9 that when the distance between the front earth electrode and the driving electrode became wider, the free rotation time became longer. This result means that the deceleration electric field became weaker; as a result, the deceleration force became weaker. This is a very natural result.

Figure 10 shows the free rotation time of the charge carrier disk when the distance between the back earth electrode and the driving electrode was changed.

Figure 10 shows that when the distance between the back earth electrode and the driving electrode became narrow, the free rotation time became longer. This result means that the acceleration electric field became stronger; as a result, the acceleration force became strong. This is a very natural result, too.

\section{3) The other parts that short the free rotation time}

When the injection voltage becomes high, the free rotation time becomes short. When the width of the driving electrode becomes wide, the free rotation time becomes short. When the polarity of the injected charge to the charge carrier and the driving electrode is different and the driving voltage is high, corona discharge occurs between the charge carrier and the driving electrode.

However, those bad effects are not critical.

\section{4) The best arrangement and potential of the electret and the electrodes}

The best arrangement and potential of the electret and electrodes were decided as follows depending on the previously described experimental results.

1) The fro nt earth electrode was removed from the equipment.

2) The distance between the driving electrode and the back earth electrode was shortened to $7 \mathrm{~mm}$.

3) The PET film that was charged to $+1.0 \mathrm{kV}-+2.0 \mathrm{kV}$ was used as the injection electret.

4) A high voltage electrode with a width of $5 \mathrm{~mm}$ was used as the driving electrode. A high-potential electret with a width of $5 \mathrm{~mm}$ has not yet been realized.

Figure 11 shows the experimental result with this improved equipment.

In this experiment, the potential of the injection electret was $+1.73 \mathrm{kV}$, and the voltage of the driving electrode was $-5.0 \mathrm{kV}$. 


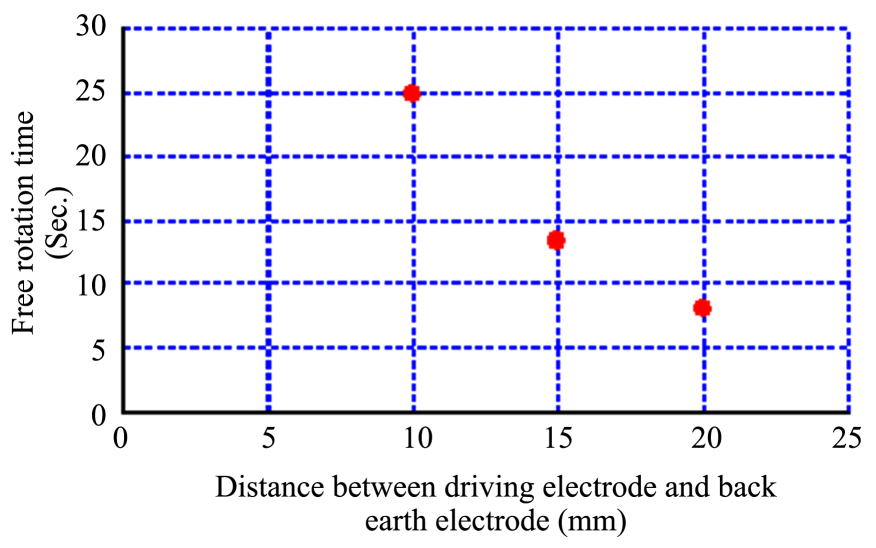

Figure 10. Free rotation time of the charge carrier disk when the distance between the back earth electrode and the driving electrode was changed.

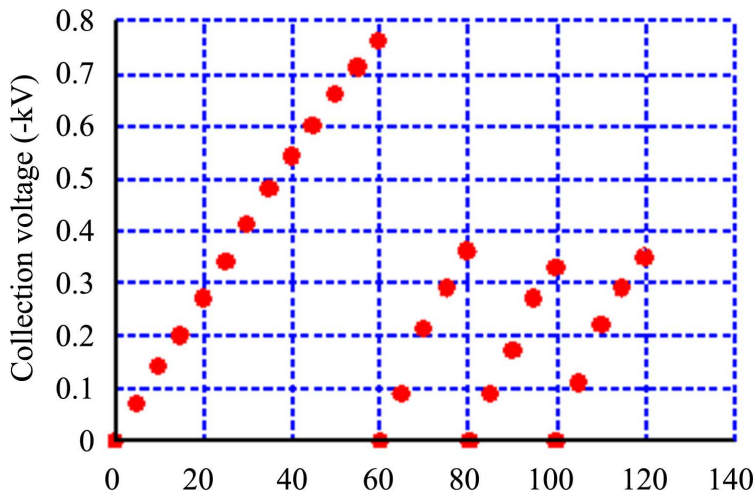

Charge carrier disk rotation time (Sec.)

Figure 11. Charge carrier disk rotation time and collection voltage.

At first, the charge carrier disk started to rotate slowly by a soft air blow. Then, the rotation speed gradually increased, and finally, the rotation speed became constant. This movement can be recognized by You Tube [12].

When the collection voltage is $-0.7 \mathrm{kV}(-700 \mathrm{~V})$, the voltage changes by 10 volts per second, and the capacitance of the collection capacitor is $1100 \mathrm{pF}$. As a result, the current becomes $-11 \mathrm{nA}$. Then, the electric power becomes $7.7 \mu \mathrm{W}$. However, theoretically, it is expected that the electric output will increase one million times.

Many (several tens) experiments were performed using this improved equipment, and the charge carrier disk rotated continuously. Namely, the experiment reappearance rate becomes $100 \%$.

When one pair of driving electrodes and earth electrodes was added to this equipment, the required driving voltage decreased from $-5.0 \mathrm{kV}$ to $-3.5 \mathrm{kV}$.

\section{Consideration}

The resolution method is a very simple idea; however, it has not been tried for a long time. The following physical Formula (2) has been considered: 


$$
\frac{1}{2} m V^{2}=F \times s
$$

where $F$ : Electrostatic force that acts on a material.

$s$ : Moving distance of the material;

$m$ : Weight of the material;

$V$ : Velocity of the material.

When force $f(\mathrm{~N})$ acts on a material with weight $m(\mathrm{~kg})$ to move its distance $s$ $(\mathrm{m})$, the velocity of the material becomes $V(\mathrm{~m} / \mathrm{s})$. If this force is an electrostatic force, the intensity is proportional to the intensity of the electric field. The intensity of the electric field is inversely proportional to the distance between the driving electrode and the earth electrode. Therefore, when the distance is shortened to $1 / 2$, the intensity of the electric field becomes two times greater. However, the material transfer distance is shortened to $1 / 2$. As a result, the velocity of the charged material is the same.

This is the reason that the distance between the two electrodes has not been changed for a long time.

This formula can be applied when the three electrodes a re placed on a straight line. However, when the three electrodes are placed on the circle, this formula will not be applied.

\section{Conclusions}

The experiment of the new electrostatic generator that was driven by an electric field succeeded. However, the success rate of the experiment was rare; therefore, the cause needed to search. Then, this problem was solved by the new arrangement of the electret and the electrode of this experimental equipment. This new arrangement can increase the acceleration force and decrease the deceleration force that both act on the charge carrier.

Today, the narrow width and high potential electret as the driving electret has not yet been realized. However, it will come true soon, and the new electrostatic generator driven by an electric field of an electret will only need to be completed.

After that, a great increase in the electric output can be attempted by new ideas.

\section{Conflicts of Interest}

The author declares no conflicts of interest regarding the publication of this paper.

\section{References}

[1] Halliday, D., Resnick, R. and Walker, J. (2002) Chapter 22 "Electric Charge" Question 1. In: Fundamentals of Physics, 6th Edition, Japanese Version, Wiley \& sons. Inc., 13.

[2] Sakai, K., et al. (2010) Chapter 1: Asymmetric Electrostatic Force and a New Electrostatic Generator. In: Bertrand, C.L., Ed., Electrostatics: Theory and Applications, 
Nova Science Publish, New York.

[3] Sakai, K. (2010) Asymmetric Electrostatic Forces and a New Electrostatic Generator. Nova Science Publish, New York.

[4] Sakai, K. (2009) The Electrostatic Force that Acts on the Charged Asymmetric Conductor in a High Electric Field. Proceedings of 2009 Electrostatics Joint Conference, $\mathrm{P} 2.08$.

[5] Sakai, K. (2010) Electrostatic Force that Acts on Non-Sphere Shape Charged Conductors. Proceedings of 2010 ESA Annual Conference, G4.

[6] Sakai, K. (2011) A Simple Experiment Result that Confirmed Asymmetric Electrostatic Force. Proceedings of 2011 ESA Annual Conference, B4.

[7] Sakai, K. (2014) Asymmetric Electrostatic Force. Journal of Electromagnetic Analysis and Applications, 6, 253-268. https://doi.org/10.4236/jemaa.2014.610026

[8] Sakai, K. (2017) Theory of Asymmetric Electrostatic Force. Journal of Electromagnetic Analysis and Applications, 9, 34-42. https://doi.org/10.4236/jemaa.2017.92004

[9] Sakai, K. (2017) The Third Trial for the New Electrostatic Generator that Is Driven by Asymmetric Electrostatic Force. Proceedings of 2017 ESA Annual Conference, A3.

[10] Sakai, K. (2019) The Electric Field Driven Generator. Global Journal of Computer Science Technology: C, 19, 1-15.

[11] Sakai, K. (2019) A New Charge Injection Method of the Electric Field Driven Generator. Proceedings of 2019 ESA Annual Conference, A4.

[12] YouTube. https://youtu.be/0eDCpgoCvIU 